\title{
Structures of Mycobacterium tuberculosis Penicillin-Binding Protein 3 in Complex with Five $\beta$-Lactam Antibiotics Reveal Mechanism of Inactivation $\$$
}

\author{
Zuokun Lu, Han Wang, Aili Zhang, Xiang Liu, Weihong Zhou, Cheng Yang, Luke Guddat, \\ Haitao Yang, Christopher J. Schofield, and Zihe Rao \\ Key Laboratory of Biomarker Based Rapid-detection Technology for Food Safety of Henan Province, Xuchang University, \\ Xuchang, Henan, China (Z.L., A.Z.); College of Life Sciences (Z.L., H.W., W.Z. and Z.R.) and College of Pharmacy (X.L., C.Y.), \\ Nankai University, Tianjin, China; School of Chemistry and Molecular Biosciences, The University of Queensland, St. Lucia, \\ Queensland, Australia (L.G.); School of Life Sciences, Tianjin University, Tianjin, China (H.Y.); University of Oxford, Chemistry \\ Research Laboratory, Oxford, United Kingdom (C.J.S.); National Laboratory of Macromolecules, Institute of Biophysics, Chinese \\ Academy of Science, Beijing, China (Z.R.); and Laboratory of Structural Biology, School of Medicine, Tsinghua University, \\ Beijing, China (Z.R.)
}

Received August 7, 2019; accepted January 14, 2020

\begin{abstract}
Because of $\beta$-lactamase-mediated resistance, $\beta$-lactam antibiotics were long considered ineffective drugs for tuberculosis (TB) treatment. However, some $\beta$-lactams, including meropenem and faropenem, are being re-evaluated in patients infected with TB. Penicillin-binding protein (PBP) 3 , or ftsl, is an essential transpeptidase in Mycobacterium tuberculosis (Mtb) required for cell division, and thus it is an important drug target. Structures of apo $M$ tbPBP3 and of complexes with five $\beta$-lactams, including meropenem and faropenem, reveal how they cause inactivation via formation of hydrolytically stable acyl-enzyme complexes. The structures reveal unique features of the antibiotic interactions, both in terms of differences in their binding to MtbPBP3 and in comparison with structures of other PBPs and serine $\beta$-lactamases, including the tautomerization status
\end{abstract}

of the carbapenem-derived acyl-enzyme complexes. The results suggest that rather than hoping PBP inhibitors developed for other infections will work against TB, work should focus on developing PBP inhibitors specialized for treating TB.

\section{SIGNIFICANCE STATEMENT}

The structures of Mycobacterium tuberculosis penicillin-binding protein 3 , an essential protein in $M$. tuberculosis, in complex with a number of widely used $\beta$-lactam antibiotics (e.g., meropenem, aztreonam, and amoxicillin) were solved. These data provide new insights for next-generation rational approaches to design tuberculosis (TB)-specific $\beta$-lactam or nonlactam antibiotics. This manuscript is a seminal article in the field of anti-TB drug discovery and suitable for the broad readership.

\section{Introduction}

In 2016, it was estimated that there were $>10$ million new cases of human tuberculosis (TB), resulting in 1.3 million deaths (World Health Organization, 2018). Thus, TB is one of the most lethal infections to afflict humankind. Furthermore, multidrug-resistant (MDR) TB and extensively drug-resistant (XDR) TB pose an increasing risk, which may result in fatalities; $<50 \%$ of patients diagnosed with MDR TB were successfully treated in 2013 , with this rate falling to $22 \%$ in patients with XDR TB (World Health Organization, 2018).

This work was supported by grants from the National Key Research and Development Program of China (Grant 15 2017YFC0840300), the Strategic Priority Research Program of the Chinese Academy of Sciences (Grant XDB08020200), the National Natural Science Foundation of China (Grant 81520108019), and the Science and Technology Planning Project of Henan Province of China (Grant 182102310071).

https://doi.org/10.1124/mol.119.118042.

S This article has supplemental material available at molpharm. aspetjournals.org.
The spread of antibacterial resistance (Cantas et al., 2013) coupled with side effects associated with the current multidrug TB treatment regimes mean the need to develop new anti-TB agents is of growing importance. For this reason, a better knowledge of the mechanisms of drug resistance and discovering new targets will aid the development of new antiTB drugs. Bedaquiline, the first new drug approved in over 40 years, targets the ATP synthase (Kundu et al., 2016). $N$-Adamantan-2-yl- $N$-((E)-3,7-dimethyl-octa-2,6-dienyl)-ethane1,2-diamine, a small molecule used for the treatment of TB under clinical phase $2 \mathrm{~b}$, is shown to bind the membrane transporter mycobacterial membrane protein Large 3 of mycobacterium (Zhang et al., 2019).

The clinical application of naturally derived $\beta$-lactams in the mid-20th century revolutionized antibacterial therapyremarkably, after 70 years, they remain the most widely used and important antibiotics. However, because of the presence of the BlaC gene in $M t b$, which encodes a highly

ABBREVIATIONS: MDR, multidrug-resistant; Mtb, Mycobacterium tuberculosis; Pa, Pseudomonas aeruginosa; PBP, penicillin-binding protein; PDB, Protein Data Bank; TB, tuberculosis; XDR, extensively drug-resistant. 
active nucleophilic serine $\beta$-lactamase, many $\beta$-lactams can be readily hydrolyzed, resulting in little or no therapeutic value (Flores et al., 2005). The BlaC $\beta$-lactamase in $M t b$ is an extended-spectrum $\beta$-lactamase with high levels of penicillinase and cephalosporinase activity as well as weak carbapenemase activity (Hugonnet and Blanchard, 2007). However, the potency of most $\beta$-lactam antibiotic classes can be restored by coadministration with a $\beta$-lactamase inhibitor, such as clavulanate, or by side chain modification. Recent studies have shown that the combination of the carbapenem meropenem and clavulanate not only has potent activity against the $H 37 \mathrm{Rv}$ strain of $M t b$ in vitro (Hugonnet et al., 2009) but is also effective in patients with XDR TB, with 6 of 7 patients on a salvage regimen containing meropenem-clavulanate showing a reduced burden of infection and one patient completely cured of the disease (Payen et al., 2012). Faropenem, the only clinically used penem, has also been shown to efficiently kill $M t b$ in the absence of a $\beta$-lactamase inhibitor (Dhar et al., 2015), and a phase 2 clinical trial is presently underway involving a combination of faropenem plus amoxicillin/clavulanate in patients with pulmonary tuberculosis (http://clinicaltrials. gov/ct2/show/NCT02349841).

The $\beta$-lactam antibiotics act as mechanism-based inhibitors by targeting the cell wall modifying $\mathrm{DD}^{\text {-transpeptidases }}$ known as penicillin-binding proteins (PBPs); in contrast to their substrates, which form acyl-enzyme complexes that are susceptible to nucleophilic attack, $\beta$-lactams react with $\mathrm{PBPs}$ to form long-lived acylated complexes. PBPs are responsible for the formation and integrity of the rigid mesh-like peptidoglycan layer exterior to the membrane surface. The most important mechanism of resistance to $\beta$-lactams is due to $\beta$-lactamases. Interestingly, $\beta$-lactamases likely evolved from a $\mathrm{PBP} / \mathrm{DD}$-peptidase-like ancestor; as a result, the active sites of both are related not only in terms of their use of a nucleophilic serine but also with respect to general acid-base catalysis and substrate-binding elements (Pratt, 2016).

MtbPBP3, a DD-transpeptidase, also known as the celldivision protein ftsI, is an essential protein that localizes at the septum, where it forms a ternary complex with other celldivision proteins ( Mukherjee et al., 2009; Plocinska et al., 2014). Inhibition of $M t b P B P 3$ by $\beta$-lactam antibiotics results in the formation of filamentous cells and the inability to undertake replication (Slayden and Belisle, 2009). Work to develop new $\beta$-lactam-based MtbPBP3 inhibitors has been hindered by a lack of structural information. Here, we describe crystal structures of the soluble region of $M t b P B P 3$ both as the apo enzyme and in complex with five antibiotics (Fig. 1), including meropenem and faropenem (data collection and refinement statistics are in Supplemental Table 1). These results advance our knowledge on the mechanisms of action of these $\beta$-lactam antibiotics and provide a platform for the direction of the design of new MtbPBP3 inhibitors.

\section{Materials and Methods}

Chemicals. Meropenem ( $>98 \%$ pure), faropenem ( $>95 \%$ pure), ampicillin ( $>95 \%$ pure), amoxicillin ( $>99 \%$ pure), and aztreonam ( $>98 \%$ pure) were purchased from TCI, Solarbio, Damas-beta, and SPC-scientific, respectively.

Cloning, Expression, and Purification. The gene encoding for $M t b$ PBP3 (spanning residue Gly123 to the end of open reading frame) was amplified by polymerase chain reaction from Mycobacterium tuberculosis H37Rv genomic DNA. The resulting polymerase chain reaction product was digested with $\mathrm{BamHI}$ and $\mathrm{XhoI}$ and inserted into the pGEX-6p-1 expression vector. Escherichia coli BL21 (DE3) cells were transformed with the recombinant plasmid, and the cells were cultured in $\mathrm{LB}$ broth at $37^{\circ} \mathrm{C}$ containing $100 \mathrm{mg} / \mathrm{l}$ ampicillin. Recombinant protein production was induced by adding isopropyl $\beta$-D-1-thiogalactopyranoside at a final concentration of $0.5 \mathrm{mM}$ at $16^{\circ}$ C. After incubation for a further 16 hours, cells were harvested by centrifugation (15 minutes at $5000 \mathrm{rpm}$ ) and then resuspended in PBS buffer. The cells were lysed by sonication for 20 minutes, and the crude extracts were centrifuged at 18,000 rpm for 40 minutes to remove cell debris. The supernatant was loaded onto a Glutathione Sepharose 4 Fast Flow (GE healthcare) column. After several rounds of alternate washing with PBS and PBS supplemented with $1 \mathrm{M} \mathrm{NaCl}, 100 \mu \mathrm{l}$ of PreScission Protease (10 $\mathrm{mg} / \mathrm{ml}$ ) was added to the column. After an overnight digestion, the protein was eluted by PBS and then concentrated and exchanged to buffer A (20 mM Tris) supplemented with $20 \mathrm{mM} \mathrm{NaCl}$. MtbPBP3 was then purified by anion-exchange chromatography using Hitrap Q HP and size-exclusion chromatography (Superdex 200) in buffer A containing $150 \mathrm{mM} \mathrm{NaCl}$.

Crystallization, Data Collection, and Structure Determination. Crystals of $M t b P B P 3$ were obtained by hanging-drop vapor diffusion at $20^{\circ} \mathrm{C}$ by mixing $1 \mu \mathrm{l}$ of protein solution $(9 \mathrm{mg} / \mathrm{ml})$ and $1 \mu \mathrm{l}$ of well solution containing $9 \%(w / v)$ polyethylene glycol $3350,5 \mathrm{mM}$ $\mathrm{CoCl}_{2}, 2 \mathrm{mM} \mathrm{MgCl}_{2}$, and 0.1 M HEPES, pH 7.4. For the acyl intermediate-complex structures, each compound (ampicillin, faropenem, amoxicillin, meropenem, and aztreonam) was prepared in the crystallization solution at a concentration of $5 \mathrm{mM}$. Two microliters of this solution was added to the crystallization drop. The mixture was allowed to equilibrate for 1 day. Before data collection, the crystals were cryoprotected in solution consisting of the well solution containing $20 \%$ glycerol. The crystals were then mounted using a cryoloop (Hampton Research). All data were collected using BL17U1 or BL19U1 at Shanghai Synchrotron Radiation Facility and processed using XDS (Kabsch, 2010). Initial phases for apo MtbPBP3 were solved by molecular replacement with Phaser (McCoy et al., 2007) in CCP4 (Winn et al., 2011) using the structure of PBP3 from Pseudomonas aeruginosa [Protein Data Bank (PDB): $3 \mathrm{PBN}$ ] as a search model.

The structure of $M t b$ PBP3 was completed by several rounds of model building with COOT (Emsley and Cowtan, 2004; Emsley et al., 2010) and refinement with REFMAC5 (Murshudov et al., 1997, 2011) in CCP4 and Phenix (Adams et al., 2010; Afonine et al., 2012). The geometric restraints for ampicillin and aztreonam were generated
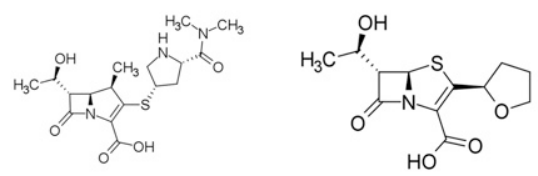

1. Meropenem
2. Faropenem

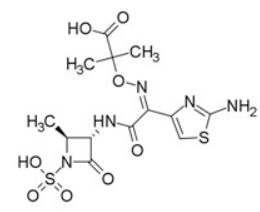

3. Aztreonam

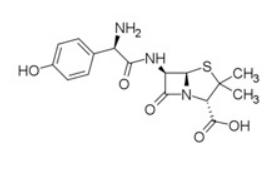

4. Amoxicillin

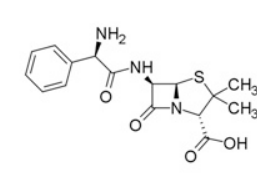

5. Ampicillin

Fig. 1. $\beta$-Lactam antibiotics used in this study. 
using the Grade Web Server (http://grade.globalphasing.org). Structure validation was carried out by MolProbity (Chen et al., 2010) in PHENIX (Adams et al., 2010). Statistics of diffraction data and refinement are shown in Supplemental Table 1.

Kinetics Assays. The methods for the kinetics assays are described in the Supplemental Information.

\section{Results}

Overall Structure. To better understand how the $\beta$-lactam antibiotics interact with an essential PBP from M. tuberculosis, we determined a crystal structure (2.1 Å resolution) of the soluble region of $M t b$ PBP3 (PDB: 6KGH) spanning residues D129 to T679 (Supplemental Table 1). This structure revealed $M t b$ PBP3 has two domains: an N-terminal domain and a highly ordered C-terminal transpeptidase domain (Fig. 2A); however, two regions in the $\mathrm{N}$-terminal domain were not visible in the electron density maps (dashed line in Fig. 2B). The function of the $\mathrm{N}$-terminal domain of class B PBPs is yet to be fully understood, but it is proposed to interact with other proteins in the divisome (Datta et al., 2006; Mukherjee et al., 2009; Sauvage et al., 2014). The MtbPBP3 transpeptidase domain comprised two subdomains, identified as $\alpha$ and $\alpha / \beta$ (yellow and blue, respectively, in Fig. 2A). Three PBP/ $\beta$-lactamase-conserved motifs (Sauvage et al., 2008; Sainsbury et al., 2011) were present in the active site, which was located at the interface between the $\alpha$ and $\alpha / \beta$ subdomains. The nucleophilic S386 and K389, which is important in acid/base catalysis, were part of the signature SXXK motif also found in $\beta$-lactamases and were located at the base of the active-site interior (Fig. 2C). The second conserved motif SXN (S441 and N443) was located on the side of the active site bordered by the $\alpha$ domain (Fig. 2C). The third conserved motif KTGT/S (K592, T593, G594, and T595) was located on the opposite active-site face and belonged to the $\alpha / \beta$ domain (Fig. 2C). The extended active-site cleft accommodated the natural polypeptide substrates, enabled catalytic crosslinking of the peptide subunits of the peptidoglycans, and was also the target site for the $\beta$-lactam antibiotics (see below).

In the apo structure, the S386 side chain pointed toward the $\alpha / \beta$ subdomain and formed a hydrogen bond with the backbone amide of T595 (2.9 $\AA$ ), but this side chain was 4.9 and 4.0 $\AA$ from K389NZ and K592NZ, respectively (Fig. 2B). The sequence and fold of the transpeptidase domain of $M t b$ PBP3 was similar to that of Pseudomonas aeruginosa ( $\mathrm{Pa}$ ) PBP3 (Han et al., 2010), with a root-mean-square deviation of $2.78 \AA$ over $294 \mathrm{C} \alpha$ atoms. Interestingly, in the PaPBP3 [PDB: 3PBN (Han et al., 2010)] structure, the hydroxyl group of the residue corresponding to S386 pointed toward the $\alpha$ subdomain, forming two hydrogen bonds to the corresponding K389 and K592 with a distance of 2.8 and $2.7 \AA$, respectively (Fig. 2D). Thus, the apo structures of $P a \mathrm{PBP} 3$ and $M t b P B P 3$ differed in this respect; the combined structures implied that induced fit involving a conformational change must occur in MtbPBP3 to enable acylation, either by a polypeptide during catalysis or by a $\beta$-lactam antibiotic during inhibition. The apparent lack of nucleophilicity for apo MtbPBP3 may reflect a means of achieving substrate selectivity.

MtbPBP3 Is Covalently Acylated by Meropenem and Faropenem. Meropenem is a broad-spectrum $\beta$-lactam antibiotic belonging to the carbapenem subgroup. Although this group of $\beta$-lactam antibiotics mimics the natural substrate of LD-transpeptidases, meropenem targets both the nucleophilic

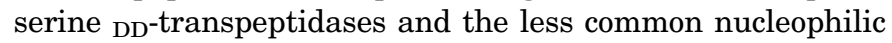

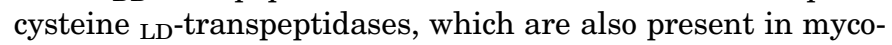
bacteria (Kohler et al., 1999; Triboulet et al., 2011; Kumar et al., 2012). Pre-steady-state kinetics analysis shows that $M t b$ PBP3 was irreversibly and fully inactivated by meropenem (Supplemental Fig. 1), with a maximum acylation rate of $k_{\text {inact }}=4.6 \pm 0.12$ minute $^{-1}$ (Supplemental Fig. 1). To visualize inactivation by meropenem, a crystal structure of the meropenem complex (PDB: 6KGS) was determined at 2.3 Å resolution. The electron density for the bond between the $\mathrm{O} \gamma$
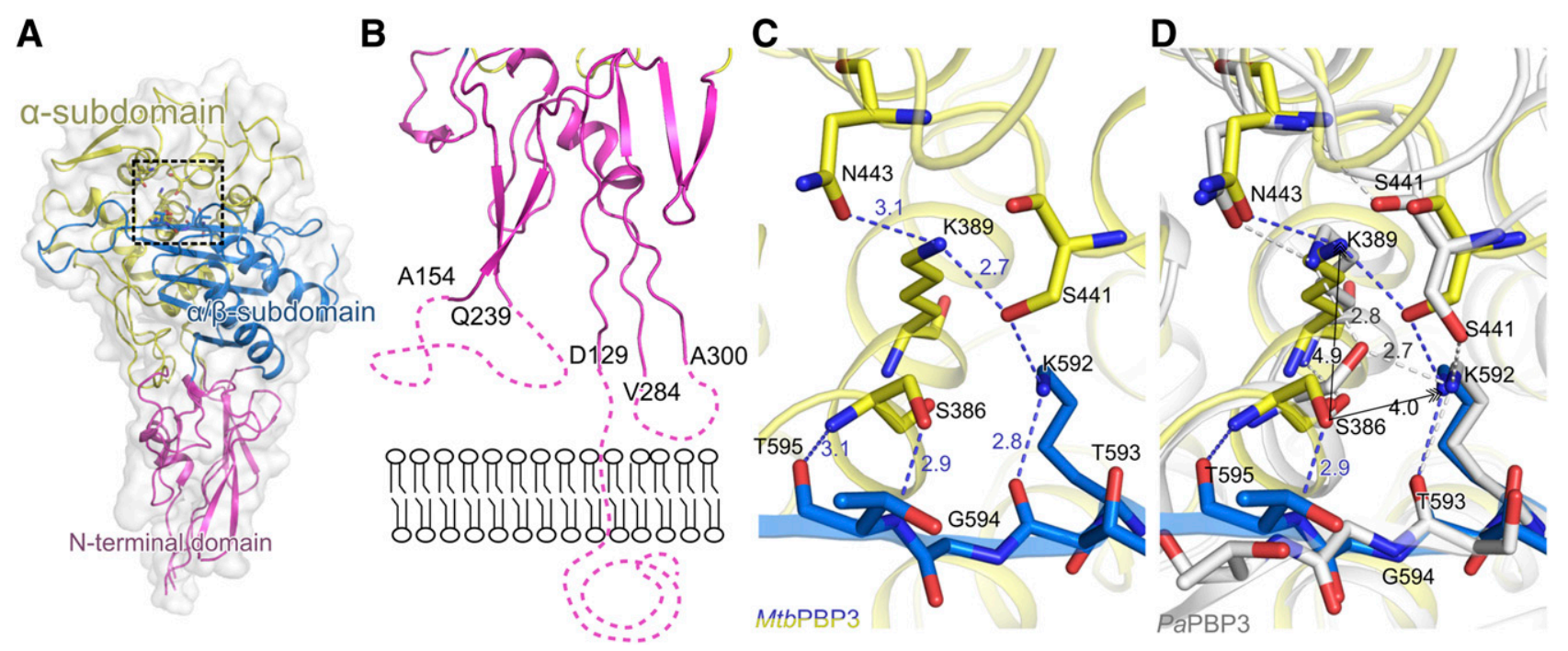

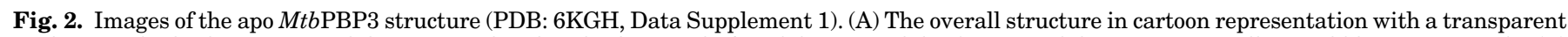

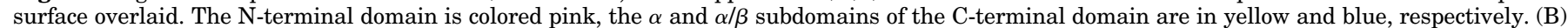

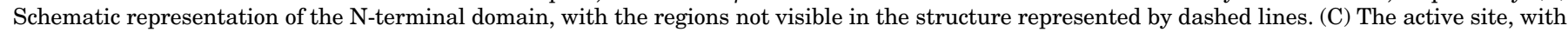

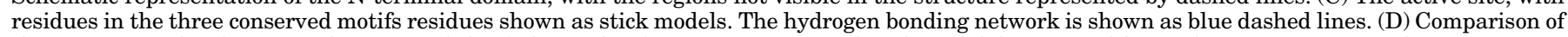

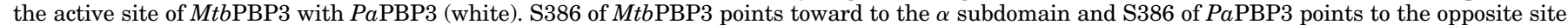

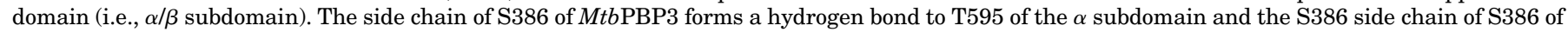

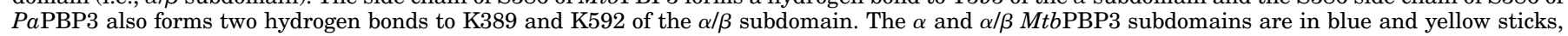
respectively. $\mathrm{PaPBP} 3$ is shown as white. 
atom of S386 and meropenem was continuous, indicating the formation of a covalent ester bond (i.e., an acyl-enzyme complex) (Fig. 3A). To make this bond, the side chain of S386 rotated by $\sim 110^{\circ}$ compared with its conformation in the apo enzyme. In this orientation, the side chains of S386 and K389 aligned parallel to each other (Fig. 3B). The S441, N443, T593, and T595 side chains also needed to adjust their conformations to accommodate meropenem (Fig. 3B), further supporting the proposal of substantial induced fit during inhibitor binding.

Critical interactions that stabilized meropenem in the active site were hydrogen bonds between: 1) the meropenemderived $\mathrm{C6}$ hydroxyethyl oxygen and the side chain of N443, 2) the meropenem-derived C3 carboxylate and the side chain of Thr593, and 3) the N4 nitrogen of the meropenem-derived pyrroline and the side chain of S441 (Fig. 3B), although hydrophobic contacts distributed throughout the active site contributed to binding. By contrast, there was a lack of stabilizing contacts between the $\mathrm{C} 2$ pyrrolidine/prolinederived ring and the enzyme. This was emphasized by the fact that the electron density for this portion of the inhibitor was not as well-resolved as the core carbapenem-derived region. Importantly, neither in the meropenem-derived structure nor any of our other MtbPBP3 inhibitor structures was there evidence for C-C fragmentation of the acyl-enzyme complexes. This contrasts sharply with the nucleophilic cyste-

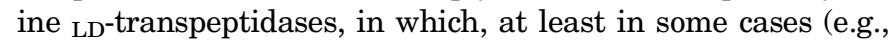
with some carbapenems and faropenem), the initially formed acyl-enzyme complexes undergo fragmentation to give hydrolytically stable species (Kumar et al., 2017). We have proposed that this difference is in part due to the differences in pKa of the ester versus thioester links in the serine DD$^{-t r a n s p e p t i d a s e s}$

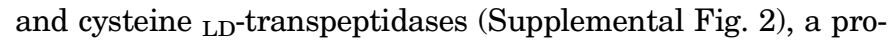
posal supported by conservation of other features between the

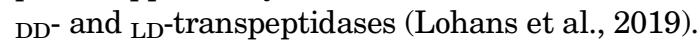

There was also a striking difference in the structures of the $M t b \mathrm{PBP} 3 \bullet$ meropenem and $M t b \mathrm{BlaC} \bullet$ meropenem complexes. In the $M t b \mathrm{BlaC} \bullet$ meropenem complex, the 2-pyrroline ring of meropenem and the initially formed acyl-enzyme complex are proposed to have undergone tautomerization, with the 2-pyrroline ring (enamine) converted to a 1-pyrroline ring (imine) (Hugonnet et al., 2009). The same configuration for meropenem was also reported in a $M t b_{\mathrm{LD}}$-transpeptidase•meropenem complex structure [PDB: 4GSU (Kim et al., 2013)]. However, in our $M t b$ PBP3•meropenem complex, the three atoms bonding to $\mathrm{C} 2$ were in a plane and thus had $\mathrm{sp}^{2}$ hybridization (i.e., enamine-imine tautomerization was not observed after the $\beta$-lactam ring opened) (Fig. 4A). This difference may reflect subtle differences in the active-site general acid/base machinery as they manifested in differences between the apo structures, including the distance between the nucleophilic oxygen and the Lys-398 N $\varepsilon$-amine. (Supplemental Figs. 3 and 4). Based on the combined structural observations, we propose (Fig. 4B) that after the $\beta$-lactam ring opened, the $\beta$-lactam ring-derived nitrogen rapidly received a proton from the hydroxyl group of S441, and the enamine tautomerization state of the pyrroline ring was retained. It is also possible that (partial) $\beta$-lactam protonation occurred prior to ring opening (Kumar et al., 2017). Note also that with some $\beta$-lactamases, S441 can act as a "secondary" nucleophile, demonstrating its potential for involvement in reaction/catalysis (Lohans et al., 2019). In $M t b \mathrm{BlaC}$, we propose that the $\mathrm{N}$ atom did not receive a proton; instead, the pyrroline ring underwent tautomerization to give an imine, possibly via an anionic intermediate, with subsequent protonation at C2 (Fig. 4C) (Page, 1984).

Faropenem is a broad-spectrum $\beta$-lactam that is active against a large number of Gram positive and Gram negative bacteria. The penems are similar to the carbapenems but with the carbon of the carbapenems being substituted by a sulfur, which is a potential leaving group. Faropenem is much more metabolically stable than meropenem and is orally available. Importantly, faropenem taken alone is more efficient than the meropenem-clavulanate combination and isoniazid in killing M. tuberculosis either in the active or latent states (Dhar et al., 2015). To investigate the interactions between $M t b P B P 3$ and faropenem, a crystal structure of the complex (PDB: 6KGT) was solved. The overall mode of binding by faropenem is similar to meropenem (Fig. 5A). A similar set of interactions stabilizing the core lactam scaffold is observed as compared with meropenem, although in the faropenem-derived complex, S441 was not positioned to form any hydrogen bonds. By contrast, the core ring of meropenem was more tightly bound by $M t b \mathrm{PBP} 3$ and the $\mathrm{N}$ atom hydrogen bonds to S441Og. Moreover, the C3 carboxylate group of meropenem was wedged between $\mathrm{S} 441$ and T593, forming two hydrogen bonds. However, in the structure of the faropenem complex, the C3 carboxylate was oriented toward the solvent, only interacting with T593. As with the pyrrolidine ring in meropenem, the tetrahydrofuran ring of faropenem was exposed to the bulk solvent and was not stabilized by any interactions to the enzyme. Its conformation also appeared to be flexible, as the electron density was relatively weak in this region.

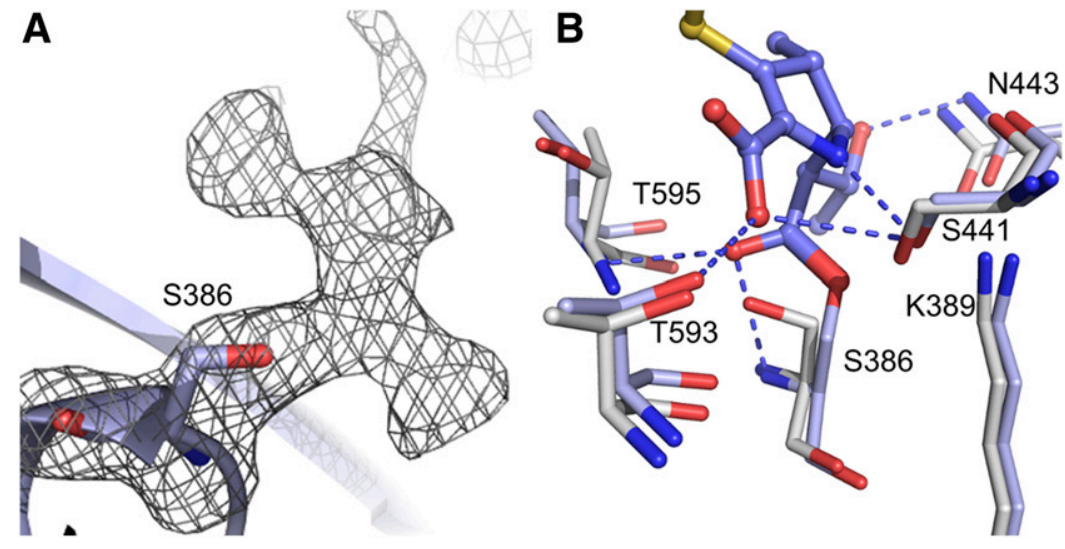

Fig. 3. $M t b P B P 3$ and interactions with meropenem (PDB: 6KGS, Data Supplement 2). (A) Omit $F_{o}-F_{c}$ electron density map (contoured at $3.0 \sigma$ ) for the region, including S386 and the binding region of meropenem. (B) The active site of the $M t b$ PBP3 acyl enzyme meropenem complex (blue) in comparison with the apo enzyme (white). 
A

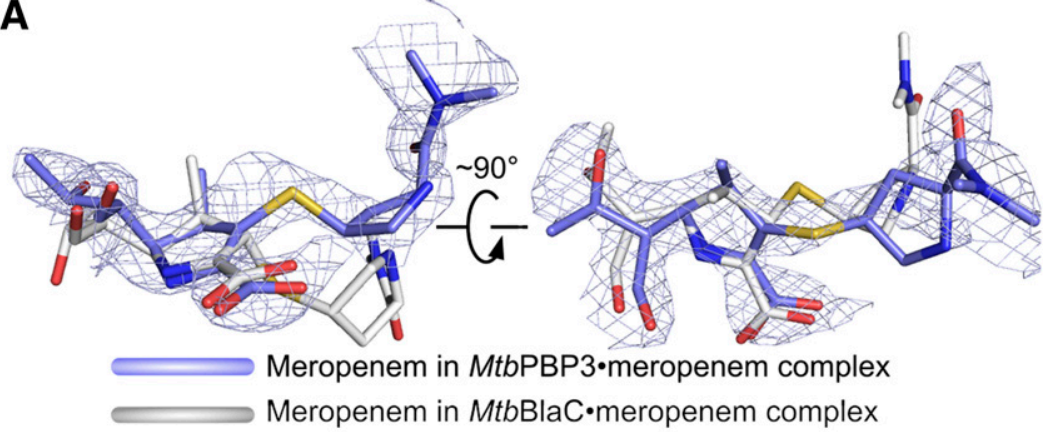

B

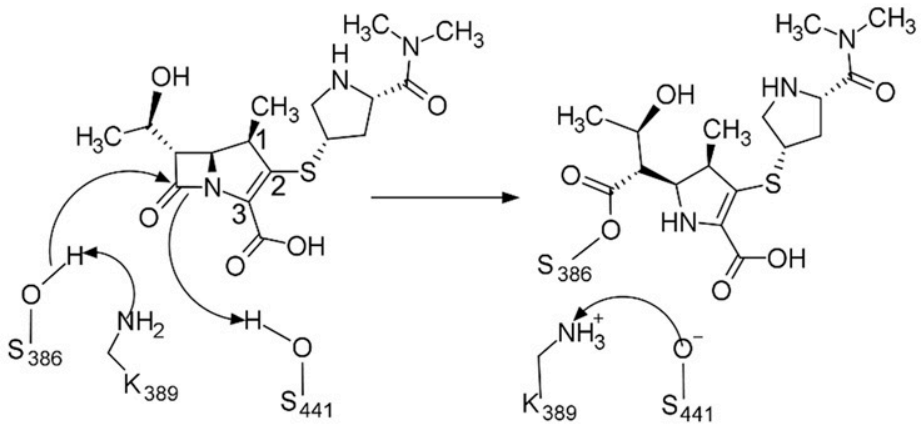

C

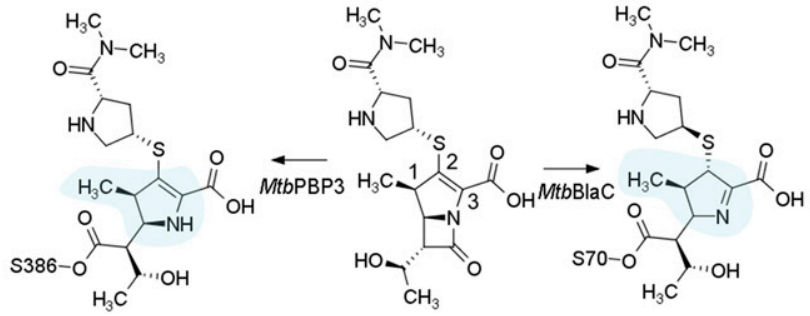

Fig. 4. Comparison of $M t b \mathrm{PBP} 3$ with $M t b \mathrm{BlaC}$. (A) Comparison of structures of meropenem in the $M t b \mathrm{PBP} 3 \bullet$ meropenem (PDB: $6 \mathrm{KGS}$ ) and $M t b \mathrm{BlaC} \bullet$ meropenem complexes (PDB: 3DWZ). (B) Proposal for the mechanism of $\beta$-lactam hydrolysis of meropenem by MtbPBP3 based on the combined crystal structures. (C) Comparison of the structures of meropenem acylating $M t b \mathrm{PBP} 3$ and $M t b \mathrm{BlaC}$.
Notably, despite the presence of the thioether group, which in other cases enables fragmentation following acyl-enzyme formation (Kumar et al., 2017; Lohans et al., 2019), no such fragmentation was observed in the $M t b P B P 3 \bullet$ faropenem complex, which is consistent with our proposal of efficient $\beta$-lactam N-protonation by MtbPBP3 (Fig. 5B).

Aztreonam Is Fully Recognized by MtbPBP3. Aztreonam is a monobactam $\beta$-lactam that is selectively active against Gram negative aerobic bacteria but exhibits little or no activity against Gram positive bacteria (Sykes et al., 1982; Brogden and Heel, 1986). In general, combination therapies involving $\beta$-lactams and clavulanate produce improved antimycobacterial activity in patients with both susceptible and MDR tuberculosis, but aztreonam was an exception (Segura et al., 1998). The lack of effectiveness of aztreonam has previously been thought to be due to weak interactions with essential PBPs found in bacteria (Sykes et al., 1982). However, our crystal structure of the $M t b P B P 3 \bullet a z t r e o n a m$ complex (PDB: 6KGU) showed the $\beta$-lactam carbonyl of aztreonam covalently bonded to the side chain of S386 in MtbPBP3, as occurs with meropenem and faropenem. In addition, as many as 13 hydrogen bonds and numerous hydrophobic interactions were formed between aztreonam and $M t b$ PBP3 (Fig. 6A). The 2-aminothiazole side chain was accommodated in a large cavity that was formed by Q501, E383, Q597, and Y606 (Fig. 6B); Q597 formed a hydrogen bond ( $3.0 \AA$ ) to the methylproponoic acid group of aztreonam. In addition, the two methyl groups of the methylpropanoic acid group were accommodated by a hydrophobic wall formed by Y611, T595, and A424; the N-sulfonate group was tightly held in place by K592, S441, T593, and T595 (Fig. 6C). Recently, work with avibactam derivatives (diazabicyclo[3.2.1] octanones) (Wang et al., 2016), bicyclic boronates (Brem et al., 2016), and structural studies, including those of noncovalent complex structures of PaPBP3 with a hydrolyzed product of cefoperazone (Ren et al., 2016), raise the possibility that non- $\beta$-lactams could be used to inhibit PBPs. The extensive interactions of MtbPBP3 with aztreonam further imply that it will be possible to design tight-binding non- $\beta$-lactam inhibitors not susceptible to $\beta$-lactamases, hence overcoming at least one mechanism of TB drug resistance.

Ampicillin and Amoxicillin Have a Similar Mode of Binding to MtbPBP3. Crystal structures of $M t b P B P 3$ in complex with the penicillin ampicillin (PDB: 6KGW) and amoxicillin (PDB: $6 \mathrm{KGV}$ ) were determined at 2.41 and $2.3 \AA$ resolution, respectively. The structures of these antibiotics are similar, except that the phenyl ring of ampicillin is substituted by a phenol in amoxicillin. The C-3 carboxylate group of ampicillin is stabilized by hydrogen bonds $(2.73$ and $2.91 \AA$ ) to the side chain hydroxyls of T593 and T595, respectively (Fig. 7A). In addition, the hydrophobic $\mathrm{C} 2$ dimethyl groups of the thiazolidine ring are held in place by the A424, W425, and T578 side chains. The $\mathrm{C} 6$ amino side chain group is held in place by hydrogen bonds with N443 and Q597 (Fig. 7B). 


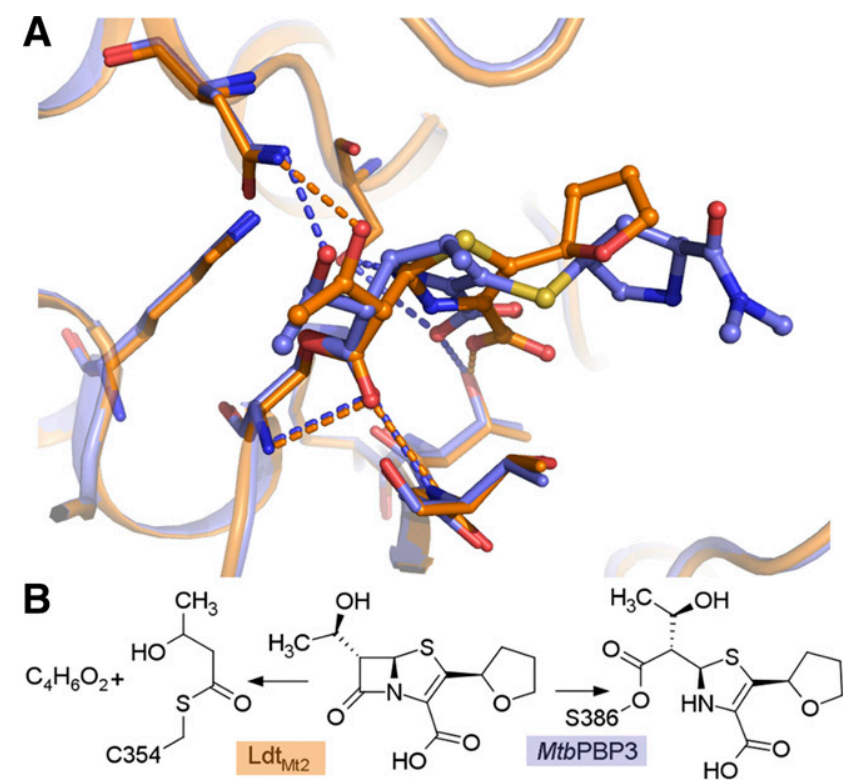

Fig. 5. MtbPBP3 and Mycobacterium tuberculosis ${ }_{\mathrm{LD}}$-transpeptidase 2 $\left(\mathrm{Ldt}_{\mathrm{Mt2}}\right)$ acylated by faropenem. (A) Comparison of structures of $M t b$ PBP3•meropenem (blue, PDB: 6KGT, Data Supplement 3) and $M t b$ PBP3•faropenem (orange). (B) Reactions of faropenem with $\mathrm{Ldt}_{\mathrm{Mt} 2}$ and $M t b P B P 3$.

\section{Discussion}

The combined crystal structures of apo MtbPBP3 and the complexes with five $\beta$-lactams (Supplemental Fig. 5) provide detailed insight into their modes of action, revealing both conserved features, such as ester formation, the role of the oxyanion hole formed by T595 and S386, and the conserved nature of acetamido/hydroxyethyl side chain-binding elements. They also reveal unique features of the antibiotic interactions in terms of differences in binding to $M t b P B P 3$ by comparison with other PBPs and serine $\beta$-lactamases, most strikingly the tautomerization status of the carbapenems.

The results support the need for future work on PBP inhibition for TB treatment. The carbapenem meropenem shows high activity against $M t b$ in vitro and in vivo (Hugonnet et al., 2009; England et al., 2012; Davies Forsman et al., 2015). Notably, the MtbBlaC $\beta$-lactamase possesses high levels of penicillinase and cephalosporinase activity but relatively weak carbapenemase activity (Hugonnet and Blanchard, 2007). In the structure of $M t b P B P 3$ in complex with meropenem, the pyrrolidine side chain meropenem is flexible and exposed to the solvent; it is thus reasonable to propose that it is not directly involved in substrate recognition. However, this moiety plays an important role in determining the spectrum of antimicrobial activity (Moellering et al., 1989; Papp-Wallace et al., 2011). Indeed, carbapenems with a C2 pyrrolidine derivative (as in meropenem) exhibit broader antimicrobial activity (Sunagawa et al., 1990). In addition to MtbBlaC, another mechanism of $\beta$-lactam resistance employed by $M t b$ involves its structurally complex cell envelope that is impermeable to many antibiotics (Fisher and Mobashery, 2016). Our structural results suggest $\mathrm{C} 2$ derivatization would be profitable both in terms of minimizing $M t b B l a C$-catalyzed hydrolysis and in optimizing permeability and efflux properties of TB targeting carbapenems.

Aztreonam is inactive against $M$. tuberculosis, with or without clavulanic acid (Segura et al., 1998). Nevertheless, our structure shows that aztreonam is recognized by $M t b \mathrm{PBP} 3$ via a binding mode involving multiple interactions, raising the possibility of using nonclassic PBP inhibitor types, including non- $\beta$-lactams (Brem et al., 2016; Wang et al., 2016) and, possibly, tight-binding noncovalent inhibitors (Ren et al., 2016) to inhibit $M t b$ PBPs. Such inhibitors warrant investigation, especially given the problems of MtbBlaC-catalyzed lactam/ acylating agent hydrolysis and localization of $M$. tuberculosis to macrophage cells.

In summary, we provided crystallographic and kinetic evidence for the mode of action of several clinically important $\beta$-lactam antibiotics targeting the essential penicillin-binging protein (PBP3) from M. tuberculosis. We show that MtbPBP3 was irreversibly and fully inactivated by meropenem, and upon being acylated, the side chain of the active serine rotated by $\sim 110^{\circ}$ to better accommodate the substrate. The overall mode of binding by faropenem is similar to meropenem, and no fragmentation was observed in the $M t b \mathrm{PBP} 3 \bullet$ faropenem complex. Aztreonam is ineffective against tuberculosis but
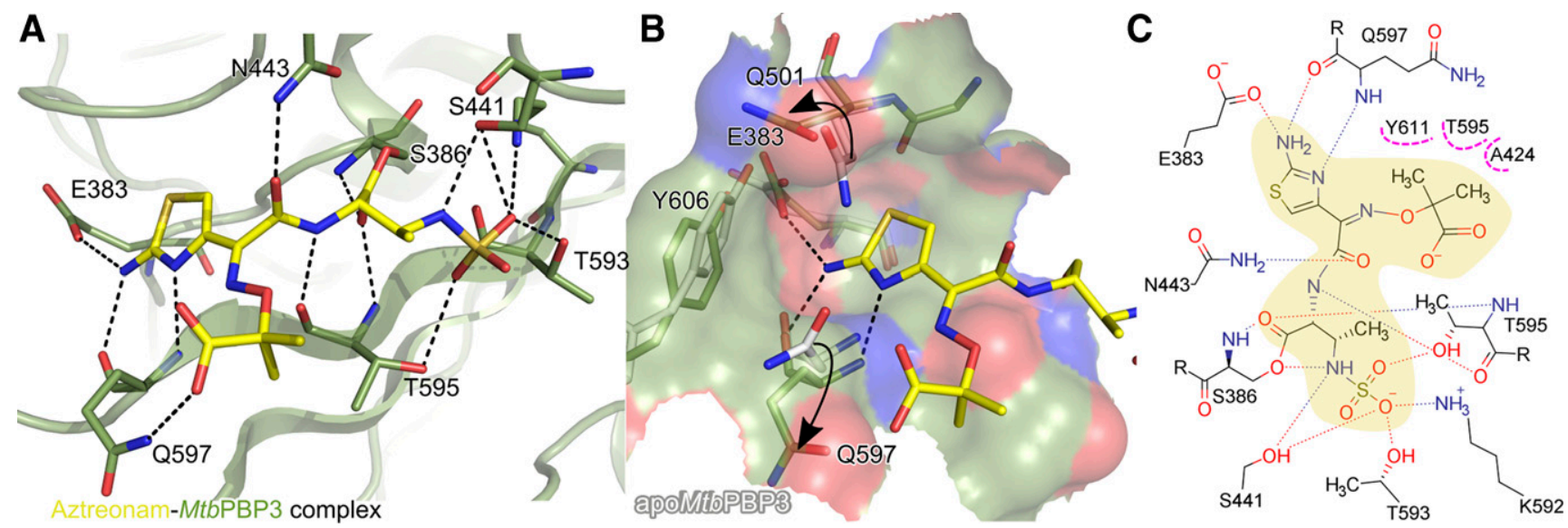

Fig. 6. Structural views of the $M t b P B P 3 \bullet a z t r e o n a m$ complex (PDB: 6 KGU, Data Supplement 4). (A) In addition to a covalent bond to S386A, a network of multiple interactions tightly binds aztreonam and MtbPBP3. (B) Surface representation for the binding mode of the aztreonam acyl-enzyme complex in the active site of $M t b$ PBP3. The aminothiazole moiety slots into a deep pocket on the surface. Key active-site residues of the apo enzyme are shown as gray sticks. The arrows represent the movement of these side chains to accommodate aztreonam. (C) Schematic of the interactions between aztreonam and MtbPBP3. Hydrogen bonds are dashed lines. Hydrophobic interactions are curved dashed lines in magenta. 

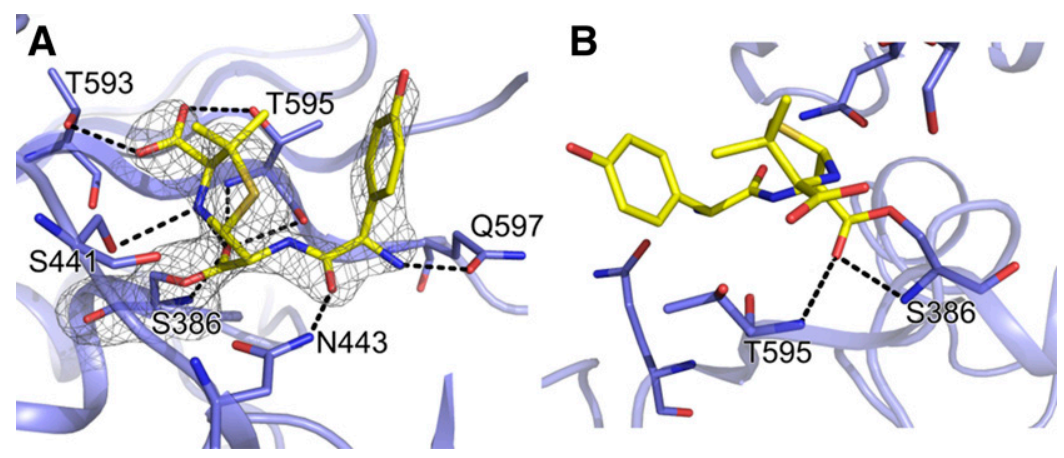

Fig. 7. The crystal structure of the $M t b P B P 3 \bullet a m o x i c i l l i n$ complex (PDB: 6KGV, Data Supplement 5). (A) Interactions of amoxicillin with $M t b$ PBP3. The $F_{o}-F_{c}$ omit map for amoxicillin and S386 is overlaid and contoured at the $3.0 \sigma$ level. (B) The carbonyl oxygen of amoxicillin is located in the oxyanion hole formed by S386, T595.

shows intact and extensive interactions with MtbPBP3, further supporting the use of non- $\beta$-lactams as PBP inhibitors. The results above suggest that rather than hoping existing PBP inhibitors developed for other infections will work against TB, work should be focused on developing PBP inhibitors specialized for TB treatment.

\section{Acknowledgments}

We thank Si Wu of the Institute of Biophysics, Chinese Academy of Science for the help of the stopped-flow experiment. We thank the staff at beamline of $17 \mathrm{U} 1$ and 19U1 of the SSRF (Shanghai Synchrotron Radiation Facility) for their assistance with data collection.

\section{Authorship Contributions}

Participated in research design: Lu, Zhang, C. Yang, H. Yang, Rao. Conducted experiments: Lu, Wang, Zhang.

Contributed new reagents or analytic tools: Lu, Zhou, H. Yang.

Performed data analysis: Lu, Liu, Zhou, C. Yang, Guddat, H. Yang, Rao.

Wrote or contributed to the writing of the manuscript: Lu, Guddat, Schofield, Rao

\section{References}

Adams PD, Afonine PV, Bunkóczi G, Chen VB, Davis IW, Echols N, Headd JJ, Hung L-W, Kapral GJ, Grosse-Kunstleve RW, et al. (2010) PHENIX: a comprehensive Python-based system for macromolecular structure solution. Acta Crystallogr D Biol Crystallogr 66:213-221.

Afonine PV, Grosse-Kunstleve RW, Echols N, Headd JJ, Moriarty NW, Mustyakimov M, Terwilliger TC, Urzhumtsev A, Zwart PH, and Adams PD (2012) Towards automated crystallographic structure refinement with phenix.refine. Acta Crystallogr D Biol Crystallogr 68:352-367.

Brem J, Cain R, Cahill S, McDonough MA, Clifton IJ, Jiménez-Castellanos JC, Avison MB, Spencer J, Fishwick CWG, and Schofield CJ (2016) Structural basis of metallo- $\beta$-lactamase, serine- $\beta$-lactamase and penicillin-binding protein inhibition by cyclic boronates. Nat Commun 7:12406.

Brogden RN and Heel RC (1986) Aztreonam. A review of its antibacterial activity, pharmacokinetic properties and therapeutic use. Drugs 31:96-130.

Cantas L, Shah SQA, Cavaco LM, Manaia CM, Walsh F, Popowska M, Garelick H, Bürgmann H, and Sørum H (2013) A brief multi-disciplinary review on antimicrobial resistance in medicine and its linkage to the global environmental microbiota. Front Microbiol 4:96.

Chen VB, Arendall WB III, Headd JJ, Keedy DA, Immormino RM, Kapral GJ, Murray LW, Richardson JS, and Richardson DC (2010) MolProbity: all-atom structure validation for macromolecular crystallography. Acta Crystallogr D Biol Crystallogr 66:12-21.

Datta P, Dasgupta A, Singh AK, Mukherjee P, Kundu M, and Basu J (2006) Interaction between $\mathrm{FtsW}$ and penicillin-binding protein 3 (PBP3) directs PBP3 to mid-cell, controls cell septation and mediates the formation of a trimeric complex involving FtsZ, FtsW and PBP3 in mycobacteria. Mol Microbiol 62:1655-1673.

Davies Forsman L, Giske CG, Bruchfeld J, Schön T, Juréen P, and Ängeby K (2015) Meropenem-clavulanate has high in vitro activity against multidrug-resistant Mycobacterium tuberculosis. Int J Mycobacteriol 4 (1):80-81.

Dhar N, Dubée V, Ballell L, Cuinet G, Hugonnet J-E, Signorino-Gelo F, Barros D, Arthur M, and McKinney JD (2015) Rapid cytolysis of Mycobacterium tuberculosis by faropenem, an orally bioavailable $\beta$-lactam antibiotic. Antimicrob Agents Chemother 59:1308-1319.

Emsley P and Cowtan K (2004) Coot: model-building tools for molecular graphics. Acta Crystallogr D Biol Crystallogr 60:2126-2132.

Emsley P, Lohkamp B, Scott WG, and Cowtan K (2010) Features and development of Coot. Acta Crystallogr D Biol Crystallogr 66:486-501.
England K, Boshoff HIM, Arora K, Weiner D, Dayao E, Schimel D, Via LE, and Barry CE III (2012) Meropenem-clavulanic acid shows activity against Mycobacterium tuberculosis in vivo. Antimicrob Agents Chemother 56:3384-3387.

Fisher JF and Mobashery S (2016) $\beta$-lactam resistance mechanisms: gram-positive bacteria and Mycobacterium tuberculosis. Cold Spring Harb Perspect Med 6: a025221.

Flores AR, Parsons LM, and Pavelka MSJ (2005) Genetic analysis of the betalactamases of Mycobacterium tuberculosis and Mycobacterium smegmatis and susceptibility to beta-lactam antibiotics. Microbiology 151:521-532.

Han S, Zaniewski RP, Marr ES, Lacey BM, Tomaras AP, Evdokimov A, Miller JR and Shanmugasundaram V (2010) Structural basis for effectiveness of siderophoreconjugated monocarbams against clinically relevant strains of Pseudomonas aeruginosa. Proc Natl Acad Sci USA 107:22002-22007.

Hugonnet JE and Blanchard JS (2007) Irreversible inhibition of the Mycobacterium tuberculosis beta-lactamase by clavulanate. Biochemistry 46:11998-12004.

Hugonnet JE, Tremblay LW, Boshoff HI, Barry CE III, and Blanchard JS (2009) Meropenem-clavulanate is effective against extensively drug-resistant Mycobacterium tuberculosis. Science 323:1215-1218.

Kabsch W (2010) XDS. Acta Crystallogr D Biol Crystallogr 66:125-132.

Kim HS, Kim J, Im HN, Yoon JY, An DR, Yoon HJ, Kim JY, Min HK, Kim S-J, Lee JY, et al. (2013) Structural basis for the inhibition of Mycobacterium tuberculosis L,D-transpeptidase by meropenem, a drug effective against extensively drugresistant strains. Acta Crystallogr D Biol Crystallogr 69:420-431.

Kohler J, Dorso KL, Young K, Hammond GG, Rosen H, Kropp H, and Silver LL (1999) In vitro activities of the potent, broad-spectrum carbapenem MK-0826 $(\mathrm{L}-749,345)$ against broad-spectrum $\beta$-lactamase-and extended-spectrum $\beta$-lactamaseproducing Klebsiella pneumoniae and Escherichia coli clinical isolates. Antimicrob Agents Chemother 43:1170-1176.

Kumar P, Arora K, Lloyd JR, Lee IY, Nair V, Fischer E, Boshoff HIM, and Barry CE III (2012) Meropenem inhibits D,D-carboxypeptidase activity in Mycobacterium tuberculosis. Mol Microbiol 86:367-381.

Kumar P, Kaushik A, Lloyd EP, Li SG, Mattoo R, Ammerman NC, Bell DT, Perryman AL, Zandi TA, Ekins S, et al. (2017) Non-classical transpeptidases yield insight into new antibacterials. Nat Chem Biol 13:54-61.

Kundu S, Biukovic G, Grüber G, and Dick T (2016) Bedaquiline targets the $\varepsilon$ subunit of mycobacterial F-ATP synthase. Antimicrob Agents Chemother 60:6977-6979.

Lohans CT, Chan HTH, Malla TR, Kumar K, Kamps JJAG, McArdle DJB, van Groesen E, de Munnik M, Tooke CL, Spencer J, et al. (2019) Non-hydrolytic $\beta$-lactam antibiotic fragmentation by l,d-transpeptidases and serine $\beta$-lactamase cysteine variants. Angew Chem Int Ed Engl 58:1990-1994.

McCoy AJ, Grosse-Kunstleve RW, Adams PD, Winn MD, Storoni LC, and Read RJ (2007) Phaser crystallographic software. J Appl Crystallogr 40:658-674.

Moellering RCJ, Eliopoulos GM, and Sentochnik DE (1989) The carbapenems: new broad spectrum beta-lactam antibiotics. J Antimicrob Chemother 24 (A):1-7.

Mukherjee P, Sureka K, Datta P, Hossain T, Barik S, Das KP, Kundu M, and Basu J (2009) Novel role of Wag31 in protection of mycobacteria under oxidative stress. Mol Microbiol 73:103-119.

Murshudov GN, Skubák P, Lebedev AA, Pannu NS, Steiner RA, Nicholls RA, Winn MD, Long F, and Vagin AA (2011) REFMAC5 for the refinement of macromolecular crystal structures. Acta Crystallogr D Biol Crystallogr 67:355-367.

Murshudov GN, Vagin AA, and Dodson EJ (1997) Refinement of macromolecular structures by the maximum-likelihood method. Acta Crystallogr D Biol Crystallogr 53:240-255.

Page MI (1984) The mechanisms of reactions of .beta.-lactam antibiotics. Acc Chem Res 17:144-151.

Papp-Wallace KM, Endimiani A, Taracila MA, and Bonomo RA (2011) Carbapenems: past, present, and future. Antimicrob Agents Chemother 55:4943-4960.

Payen MC, De Wit S, Martin C, Sergysels R, Muylle I, Van Laethem Y, and Clumeck N (2012) Clinical use of the meropenem-clavulanate combination for extensively drug-resistant tuberculosis. Int $J$ Tuberc Lung Dis 16:558-560.

Plocinska R, Martinez L, Gorla P, Pandeeti E, Sarva K, Blaszczyk E, Dziadek J, Madiraju MV, and Rajagopalan M (2014) Mycobacterium tuberculosis MtrB sensor kinase interactions with FtsI and Wag31 proteins reveal a role for MtrB distinct from that regulating MtrA activities. J Bacteriol 196:4120-4129.

Pratt RF (2016) $\beta$-Lactamases: why and how. J Med Chem 59:8207-8220.

Ren J, Nettleship JE, Males A, Stuart DI, and Owens RJ (2016) Crystal structures of penicillin-binding protein 3 in complexes with azlocillin and cefoperazone in both acylated and deacylated forms. FEBS Lett 590:288-297.

Sainsbury S, Bird L, Rao V, Shepherd SM, Stuart DI, Hunter WN, Owens RJ, and Ren J (2011) Crystal structures of penicillin-binding protein 3 from Pseudomonas aeruginosa: comparison of native and antibiotic-bound forms. J Mol Biol 405:173-184. 
Sauvage E, Derouaux A, Fraipont C, Joris M, Herman R, Rocaboy M, Schloesser M, Dumas J, Kerff F, Nguyen-Distèche M, et al. (2014) Crystal structure of penicillinbinding protein 3 (PBP3) from Escherichia coli. PLoS One 9:e98042.

Sauvage E, Kerff F, Terrak M, Ayala JA, and Charlier P (2008) The penicillin-binding proteins: structure and role in peptidoglycan biosynthesis. FEMS Microbiol Rev 32 234-258.

Segura C, Salvadó M, Collado I, Chaves J, and Coira A (1998) Contribution of betalactamases to beta-lactam susceptibilities of susceptible and multidrug-resistant Mycobacterium tuberculosis clinical isolates. Antimicrob Agents Chemother 42: 1524-1526.

Slayden RA and Belisle JT (2009) Morphological features and signature gene response elicited by inactivation of FtsI in Mycobacterium tuberculosis. J Antimicrob Chemother 63:451-457.

Sunagawa M, Matsumura H, Inoue T, Fukasawa M, and Kato M (1990) A nove carbapenem antibiotic, SM-7338 structure-activity relationships. J Antibiot (Tokyo) 43:519-532.

Sykes RB, Bonner DP, Bush K, and Georgopapadakou NH (1982) Azthreonam (SQ 26,776 ), a synthetic monobactam specifically active against aerobic gram-negative bacteria. Antimicrob Agents Chemother 21:85-92.
Triboulet S, Arthur M, Mainardi JL, Veckerlé C, Dubée V, Nguekam-Moumi A, Gutmann L, Rice LB, and Hugonnet JE (2011) Inactivation kinetics of a new target of beta-lactam antibiotics. J Biol Chem 286:22777-22784.

Wang DY, Abboud MI, Markoulides MS, Brem J, and Schofield CJ (2016) The road to avibactam: the first clinically useful non- $\beta$-lactam working somewhat like a $\beta$ lactam. Future Med Chem 8:1063-1084.

World Health Organization (2018) Global Tuberculosis Report 2018, World Health Organization, Geneva, Switzerland.

Winn MD, Ballard CC, Cowtan KD, Dodson EJ, Emsley P, Evans PR, Keegan RM Krissinel EB, Leslie AGW, McCoy A, et al. (2011) Overview of the CCP4 suite and current developments. Acta Crystallogr D Biol Crystallogr 67:235-242.

Zhang B, Li J, Yang X, Wu L, Zhang J, Yang Y, Zhao Y, Zhang L, Yang X, Yang X, et al. (2019) Crystal structures of membrane transporter MmpL3, an anti-TB drug target. Cell 176:636-648.e13.

Address correspondence to: Dr. Zihe Rao, College of Life Sciences, Nankai University, Tianjin 30071, China. E-mail: raozh@mail.tsinghua.edu.cn 